\title{
RELATÓRIO DE ATIVIDADE ACADÊMICA COMO RECURSO DE APRENDIZAGEM DA PESQUISA
}

\author{
Zeneide Soubhia ${ }^{1}$ \\ Márcia Caron Ruffino ${ }^{2}$ \\ Elma Mathias Dessunti ${ }^{1}$
}

Soubhia Z, Ruffino MC, Dessunti EM. Relatório de atividade acadêmica como recurso de aprendizagem da pesquisa. Rev Latino-am Enfermagem 2005 março-abril; 13(2):269-73.

O trabalho foi desenvolvido com o objetivo de analisar os relatórios de atividades acadêmicas quanto à estrutura e habilidades mentais desenvolvidas pelos graduandos de duas propostas curriculares do Curso de Enfermagem da Universidade Estadual de Londrina. Participaram do estudo 83 alunos; 39 do Currículo de Transição e 44 do Currículo Integrado. Os dados foram coletados por meio de uma ficha de registro que contempla os tópicos do relatório, em dezembro de 2002, dos alunos do Currículo de Transição, e em dezembro de 2003, dos alunos do Currículo Integrado. Os resultados, apresentados na forma de freqüência percentual, apontam que os alunos do Currículo Integrado elaboraram o relatório de atividade acadêmica com estrutura mais adequada que o dos alunos do Currículo de Transição. Esse grupo de graduandos também desenvolveu raciocínios mais complexos, especialmente no tópico "desenvolvimento". Conclui-se que as intervenções junto aos alunos do Currículo Integrado propiciaram melhor desenvolvimento nessa estratégia de ensino.

DESCRITORES: avaliação de desempenho; pesquisa; alunos de enfermagem; relatório; enfermagem

\section{ACADEMIC ACTIVITY REPORT AS A STRATEGY FOR LEARNING RESEARCH}

This study aimed to analyze academic activity reports about the structure and mental abilities developed by graduate students of two nursing curriculums proposals at a Brazilian university. The study was based on 83 students: 39 from the Transition Curriculum (group I) and 44 from the Integrated Curriculum (group II). Data were collected through a registration card with topics from the December 2002 report by group I and the December 2003 report by group II. The results, presented as frequency percentages, showed that group II elaborated the academic activity report with a better structure than group I. This group also developed a more complex reasoning, especially in the "development" topic. Interventions with group II present a better development in this teaching strategy.

DESCRIPTORS: performance assessment; research; students, nursing; report; nursing

\section{INFORME DE ACTIVIDAD ACADÉMICA COMO RECURSO DE APRENDIZAJE DE INVESTIGACIÓN}

La finalidad de este trabajo fue analizar los informes académicos respecto a la estructura y habilidades mentales desarrolladas por graduandos en dos propuestas curriculares del Curso de Enfermería de la Universidad Estadual de Londrina, Brasil. Participaron del estudio 83 alumnos: 39 del Currículo de Transición y 44 del Currículo Integrado. Los datos se obtuvieron a través de un formulario de registro que contempla los tópicos del informe, en Diciembre del 2002 para los alumnos del Currículo de Transición y en Diciembre del 2003 para los del Currículo Integrado. Los resultados, presentados en una lista de frecuencia porcentual, indican que los alumnos del Currículo Integrado elaboraran informes académicos con estructura más adecuada que los alumnos del Currículo de Transición. Este grupo de alumnos también desarrolló raciocinios más complexos, en especial en el tópico "desarrollo". Las intervenciones en el grupo del Currículo Integrado permitieron resultados superiores en esta estrategia de enseñanza.

DESCRIPTORES: evaluación del rendimiento; investigación; estudiantes de enfermería; informe; enfermería

\footnotetext{
${ }^{1}$ Doutor em Enfermagem, Professor da Universidade Estadual de Londrina, e-mail: zeneide@uel.br; ${ }^{2}$ Professor Titular da Escola de Enfermagem de Ribeirão Preto, da Universidade de São Paulo, Centro Colaborador da OMS para o desenvolvimento da pesquisa em enfermagem
} 


\section{INTRODUÇÃO}

Orelatório é uma atividade que tem merecido nossa atenção pelas possibilidades que apresenta para o ensino e a aprendizagem da pesquisa. Num primeiro momento, o relatório consistia em organizar as informações de vários autores, em texto único, para enriquecer os conceitos subsunçores dos diferentes temas estudados pelos alunos. $O$ conceito subsunçor, para alguns autores ${ }^{(1-3)}$, é 0 conceito que serve de ancoradouro para novas informações que vão sendo incorporadas na estrutura cognitiva do indivíduo, no decorrer da sua história, por meio dos processos de diferenciação progressiva e reconciliação integrativa. À medida que novas informaç̧̃̃es vão sendo assimiladas, 0 conceito subsunçor vai-se fortalecendo e tornando-se mais abrangente.

Atualmente, consideramos o relatório como recurso que possibilita ao aluno o exercício de várias habilidades mentais complexas como argumentar, analisar, contrapor, justificar, compor e propor; habilidades essas salientadas como necessárias à formação acadêmica do aluno ${ }^{(4)}$.

O Curso de Enfermagem da Universidade Estadual de Londrina (UEL), criado em 1972, passou por cinco reformas curriculares. Em 1996, ocorreu a quarta reforma curricular que trouxe alterações significativas como: estímulo à interdisciplinaridade, fusão do ciclo básico com o clínico, inserção do aluno nos campos de atuação do enfermeiro desde o primeiro ano do curso. Foi considerado de transição para o Currículo Integrado, para que as dificuldades pudessem ser gradativamente superadas, diminuindo-se resistências e capacitando-se docentes para as mudanças metodológicas pretendidas. Em 2000, o Curso de Enfermagem implantou a quinta reforma curricular, o Currículo Integrado, proposta que encerra a divisão dos conteúdos por disciplina e utiliza a Pedagogia da Problematização como principal recurso de ensino.

O Currículo Integrado está organizado por áreas de conhecimento, consideradas "seivas", que permeiam todos os módulos: comunicação, bioética, trabalho em equipe, metodologia de assistência e metodologia de pesquisa ${ }^{(5)}$.

Nessa proposta, o papel do aluno é de sujeito ativo no processo de construção do seu próprio conhecimento ${ }^{(6)}$. O papel do professor é orientar o processo de aprendizagem, mediante a promoção da aprendizagem significativa e estimulação do raciocínio do aluno.

Nesse contexto, o relatório torna-se um recurso importante de aprendizagem, pois ele representa uma maneira de o aluno elaborar a síntese dos conhecimentos construídos por ele, a partir da busca de informações em várias áreas de conhecimento.

O relatório é uma atividade acadêmica que vem sendo exercitada pelos alunos, com a finalidade de desenvolver as habilidades mentais complexas, de organizar a síntese dos conhecimentos construídos nas unidades dos módulos e de desenvolver a habilidade escrita; aos poucos, o relatório aproxima-se do formato do artigo científico. As instituições de ensino estão, aos poucos, tomando consciência da importância do ensino da pesquisa na graduação $0^{(7)}$.

Essas considerações levaram-nos ao seguinte problema: Qual é o perfil dos relatórios das atividades acadêmicas dos alunos que estão cursando o último ano do Curso de Enfermagem?

O relatório de atividade acadêmica é um documento escrito no qual se descrevem, sistematicamente, os passos de desenvolvimento de um tema e das atividades junto à comunidade ou da pesquisa científica. Esse documento possui dois aspectos: apresentação e estrutura. Aapresentação refere-se ao seu aspecto externo, ao visual; a estrutura diz respeito à distribuição das idéias nos tópicos. Vários autores dividem a estrutura dos documentos científicos em elementos pré- textuais, textuais e pós- textuais. São elementos pré-textuais: capa, folha de rosto e anverso da folha de rosto; elementos textuais: introdução, desenvolvimento e conclusão; e pós- textuais: referências, anexo e glossário ${ }^{(8-10)}$.

Acreditamos que o relatório, além das características já descritas, é uma obra de arte, e procuramos incentivar os alunos com essa idéia. É arte porque é resultado de outras atividades como o é 0 estudo de textos, os quais devem ser compreendidos em sua totalidade, deles extraindo-se as idéias principais que serão usadas como ferramentas para compreender a situação em estudo e justificar condutas. É ato criativo porque é capaz de desencadear outros atos criadores $^{(11)}$.

O relatório é um recurso importante na formação do aluno em pesquisa, porquanto favorece o desenvolvimento de habilidades em leitura, de conceitos e técnicas relacionados ao trabalho científico, na análise de artigos e produção de textos.

Este tema foi desenvolvido com os seguintes objetivos: analisar os relatórios de graduandos em enfermagem, quanto à estrutura, distribuição das idéias nos tópicos e habilidades mentais desenvolvidas; comparar os desempenhos dos formandos de duas propostas curriculares na elaboração do relatório de atividades acadêmicas.

\section{MATERIAL E MÉTODO}

Este estudo foi desenvolvido com os relatórios produzidos por formandos de duas propostas curriculares do Curso de Graduação em Enfermagem da UEL. Foram analisados 39 relatórios dos alunos do Currículo de Transição (C.T.) e 44 dos alunos do Currículo Integrado (C.I).

O instrumento de registro dos dados do relatório constituiuse de uma ficha composta por cinco tópicos: título, introdução, 
desenvolvimento, conclusão e referências. A coleta dos dados foi realizada no final de dezembro de 2002, para um grupo, e no final de dezembro de 2003, para outro grupo. Analisamos o relatório por meio de duas leituras: na primeira, procuramos tomar conhecimento do conteúdo do trabalho e, na segunda, utilizamos o grifo e as anotações à margem das páginas, para identificar as idéias de cada tópico.

Os dados foram organizados em uma tabela de freqüência que apresenta os dados brutos, a proporção de acertos e o grau de significância. Para a comparação dos desempenhos entre os dois grupos, utilizamos o teste de Mann-Whitney $\left(\mathrm{x}^{2}\right)$ ou o teste de Fisher.
O projeto que originou este trabalho foi aprovado pelo Comitê de Ética do Hospital Universitário Regional Norte do Paraná, e todos os alunos assinaram o termo de consentimento livre e esclarecido.

\section{RESULTADOS}

Os dados referentes aos relatórios dos graduandos do Curso de Enfermagem estão apresentados na Tabela 1 e seguem a ordem dos tópicos de um relatório de atividade acadêmica.

Tabela 1 - Distribuição dos acertos na estrutura do relatório de atividade acadêmica pelos graduandos de Enfermagem, UEL, 2004

\begin{tabular}{|c|c|c|c|c|c|c|c|c|}
\hline \multirow{2}{*}{ 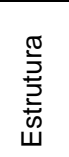 } & \multirow[t]{2}{*}{ Idéias básicas } & \multicolumn{2}{|c|}{$\begin{array}{l}\text { Currículo Transição } \\
n=39\end{array}$} & \multicolumn{2}{|c|}{$\begin{array}{c}\text { Currículo Integrado } \\
n=44\end{array}$} & \multirow[t]{2}{*}{ qui } & \multirow[t]{2}{*}{ gl } & \multirow[t]{2}{*}{$\mathrm{P}$} \\
\hline & & sujeitos & $\%$ & Sujeitos & $\%$ & & & \\
\hline \multirow{7}{*}{$\stackrel{\text { 을 }}{\underline{E}}$} & Claro & 14 & 35,9 & 43 & 97,7 & 33,92 & 1 & $<0,0001$ \\
\hline & Conciso & 11 & 28,2 & 43 & 97,7 & 40,95 & 1 & $<0,0001$ \\
\hline & Concreto & 11 & 28,2 & 43 & 97,7 & 40,95 & 1 & $<0,0001$ \\
\hline & Criativo & 8 & 20,5 & 13 & 29,5 & 0,48 & 1 & 0,4891 \\
\hline & Apresenta-se título/tema & 20 & 51,3 & 33 & 75,0 & 4,06 & 1 & 0,0438 \\
\hline & Definem-se termos/conceitos & 17 & 43,6 & 27 & 61,4 & 1,96 & 1 & 0,1618 \\
\hline & Delimita-se o problema & 7 & 17,9 & 28 & 63,6 & 15,87 & 1 & $<0,0001$ \\
\hline \multirow{9}{*}{ 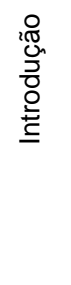 } & Faz justificativa & 11 & 28,2 & 27 & 61,4 & 7,87 & 1 & 0,0050 \\
\hline & Pessoal & 4 & 10,3 & 16 & 36,4 & 6,34 & 1 & 0,0118 \\
\hline & Profissional & 5 & 12,8 & 27 & 61,4 & 18,57 & 1 & $<0,0001$ \\
\hline & Realidade & 4 & 10,3 & 25 & 56,8 & 17,72 & 1 & $<0,0001$ \\
\hline & Como considerado $\mathrm{p} /$ autores & 11 & 28,2 & 16 & 36,4 & 0,31 & 1 & 0,5775 \\
\hline & Apresenta desenvolvimento & 14 & 35,9 & 24 & 54,5 & 2,19 & 1 & 0,1386 \\
\hline & Faz estimulação & 1 & 2,6 & 4 & 9,1 & Fisher & & 0,3642 \\
\hline & Definem-se objetivos & 22 & 56,4 & 38 & 86,4 & 7,83 & 1 & 0,0052 \\
\hline & Define & 13 & 33,3 & 33 & 75,0 & 12,89 & 1 & 0,0003 \\
\hline \multirow{8}{*}{ 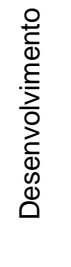 } & Descreve & 33 & 84,6 & 42 & 95,5 & Fisher & & 0,1394 \\
\hline & Compara autores & 13 & 33,3 & 5 & 11,4 & 4,65 & 1 & 0,0310 \\
\hline & Justifica & 6 & 15,4 & 37 & 84,1 & 36,39 & 1 & $<0,0001$ \\
\hline & Analisa & 18 & 46,2 & 38 & 86,4 & 13,45 & 1 & 0,0002 \\
\hline & Julga & 17 & 43,6 & 38 & 86,4 & 15,06 & 1 & 0,0001 \\
\hline & Critica & 15 & 38,5 & 36 & 81,8 & 14,63 & 1 & 0,0001 \\
\hline & Propõe & 14 & 35,9 & 35 & 79,5 & 14,53 & 1 & 0,0001 \\
\hline & Relaciona teoria e prática & 30 & 76,9 & 39 & 88,6 & 1,27 & 1 & 0,2591 \\
\hline \multirow{6}{*}{ 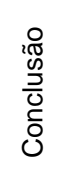 } & Sintetiza & 13 & 31,7 & 41 & 93,2 & 30,00 & 1 & $<0,0001$ \\
\hline & Relaciona com objetivos & 5 & 12,2 & 23 & 52,3 & 12,68 & 1 & 0,0004 \\
\hline & com desempenhos & 1 & 2,4 & 5 & 11,4 & Fisher & & 0,2067 \\
\hline & com papel do enfermeiro & 3 & 7,3 & 18 & 40,9 & 10,38 & 1 & 0,0013 \\
\hline & Fecha raciocínios desenv. & 26 & 63,4 & 36 & 81,8 & 1,77 & 1 & 0,1829 \\
\hline & Sugere & 6 & 14,6 & 23 & 52,3 & 10,81 & 1 & 0,0010 \\
\hline \multirow{6}{*}{ 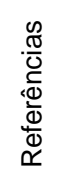 } & Faz citações no texto & 25 & 64,1 & 38 & 86,4 & 4,45 & 1 & 0,0349 \\
\hline & Coerentes com citações & 24 & 61,5 & 25 & 56,8 & 0,05 & 1 & 0,8315 \\
\hline & com normas da ABNT & 25 & 64,1 & 40 & 90,9 & 7,24 & 1 & 0,0071 \\
\hline & Pertinentes & 30 & 76,9 & 40 & 90,9 & 2,09 & 1 & 0,1478 \\
\hline & Atualizadas & 30 & 76,9 & 40 & 90,9 & 2,09 & 1 & 0,1478 \\
\hline & Especificas & 30 & 76,9 & 40 & 90,9 & 2,09 & 1 & 0,1478 \\
\hline Total & 36 & 537 & 38,3 & 1079 & 68,1 & 266,22 & 1 & $<0,0001$ \\
\hline
\end{tabular}

Os dados apresentados na Tabela 1 indicam que 0 desempenho geral na elaboração do relatório foi melhor entre os alunos do Currículo Integrado, com diferença significante também na análise da maioria das partes que o compõem.

\section{DISCUSSÃO}

Em relação ao título, os alunos do C.I. obtiveram melhores resultados, com diferença significante em três das quatro variáveis consideradas (clareza, concisão e concretude). A característica criatividade foi a menos utilizada pelos dois grupos, merecendo ser salientada nos momentos de orientação da atividade. Os títulos de trabalhos científicos devem possuir as características citadas, pois eles revelam atributos da personalidade dos autores; portanto, é aconselhável que se dispense o tempo necessário para a elaboração desse aspecto do trabalho ${ }^{(12)}$.

Na introdução, os conceitos mais desenvolvidos pelos dois 
grupos dizem respeito à "apresentação do tema" e a "objetivos", com diferença significante nos resultados em favor dos alunos do C.I. Esses alunos ainda apresentam melhores desempenhos na "delimitação do problema" e na "justificativa".

No desenvolvimento, a "descrição" foi o recurso mais explorado pelos dois grupos. Quanto à "relação entre a teoria e a prática", não houve diferença significante entre os grupos. Os alunos do C.I. apresentam resultados superiores, com diferença significante na maioria dos itens, demonstrando melhor habilidade nessa etapa do relatório. Embora os alunos do C.T. tenham tido melhor desempenho na "comparação das idéias dos autores", percebemos que poucos utilizaram esse recurso, talvez por falta de entendimento da relevância dessa habilidade e por ser a descrição o recurso mais utilizado. Percebemos que os raciocínios mais complexos foram desenvolvidos pelos alunos do C.I. (justifica, analisa, julga, critica e propõe). Apesar da alta percentagem do resultado em "descrever," isso não significa que o aluno incorporou o objetivo do relatório, que não é espaço para cópia, imitação, reprodução, mas, sim, lugar de criatividade.

Esse tópico do relatório merece continuar sendo incentivado e orientado pelos docentes para que os alunos desenvolvam as habilidades de justificar, analisar, argumentar ${ }^{(4)}$.

Na conclusão, a habilidade de "sintetizar" e "fechar os raciocínios" foram as mais utilizadas pelos dois grupos. Os testes não indicaram diferença significante em dois itens: "relaciona com os desempenhos" " "fecha os raciocínios desenvolvidos". Percebemos que os alunos do C.I. preocuparam-se em relacionar a conclusão com os objetivos e com o papel da enfermeira. Provavelmente, isso seja conseqüência da metodologia que estimula o contato com a realidade antes da fundamentação teórica.

A conclusão dos relatórios necessita da atenção dos docentes, os quais devem orientar os alunos a relacionar os temas estudados com os desempenhos ou objetivos, com o papel da enfermeira e fechar os raciocínios desenvolvidos. A conclusão deve ser breve, clara, objetiva e fechar os raciocínios desenvolvidos em torno do problema ${ }^{(13)}$.

No tópico "referências", os dois grupos alcançaram melhores resultados quando comparados com os desempenhos obtidos nos tópicos anteriores. Parece-nos que esses dados refletem a atenção dos professores com o desenvolvimento da habilidade de organizar as referências.

Na análise global dos dados da Tabela 1, observamos que

\section{REFERÊNCIAS BIBLIOGRÁFICAS}

1. Soubhia Z. Programa educativo em estratégias de estudo. [Dissertação]. Ribeirão Preto (SP): Escola de Enfermagem de Ribeirão Preto/USP; 1990.

2. Ausubel DP, Novak JD, Hanesian H. Psicologia educacional. $2^{\mathrm{a}}$ ed. Rio de Janeiro (RJ): Interamericana; 1978. os alunos do C.I são superiores aos alunos do C.T. na maioria das variáveis dos tópicos que compõem o relatório.

Os resultados obtidos pelos alunos do C.I. refletem as mudanças do projeto pedagógico desse Currículo. Esses alunos demonstraram ter exercitado várias vezes o relatório e já haver incorporado habilidades como: estrutura, conceitos próprios de cada tópico, e também ter aprendido a valorizar a coerência das partes. 0 relatório é uma atividade complexa que requer o desenvolvimento de outras habilidades como: domínio de estratégias de estudo, habilidade de síntese e dedicação. É um produto criativo, pois a escolha de conceitos, de autores e a organização do texto são pessoais. Mas criar não é tirar do nada, e sim dar significado pessoal às idéias retiradas dos autores $^{(14)}$.

Salientamos que o relatório é uma atividade que tem sido incentivada pelo grupo de docentes do qual participamos. Com a implantação do atual currículo tivemos várias aproximações de pequenos grupos de alunos, culminando com as atividades do módulo 10 no último ano do curso, com acompanhamento de todos os alunos nas etapas de elaboração do relatório. Provavelmente, a conduta uniforme e sistematizada dos docentes em relação à elaboração dos relatórios tenha resultado no melhor desempenho desses alunos nessa atividade.

A competência na elaboração do relatório exige disciplina que só se adquire com a prática.

\section{CONCLUSÃO}

Os dados apresentados permitem-nos concluir que os títulos dos relatórios dos alunos do Currículo Integrado foram mais claros, concretos e concisos que os dos alunos do Currículo de Transição; na introdução, objetivo e apresentação do tema são aspectos mais desenvolvidos do relatório pelos dois grupos; no desenvolvimento, os raciocínios mais complexos foram mais desenvolvidos pelos alunos do Currículo Integrado; na conclusão, os dois grupos de alunos fecharam os raciocínios desenvolvidos no corpo do relatório; nas referências, os dois grupos demonstraram a compreensão de que elas devem ser atualizadas, específicas e pertinentes.

Considerando todos os tópicos do relatório, os alunos do Currículo Integrado apresentaram resultados superiores aos dos alunos do Currículo de Transição.

3. Moreira MA, Buchweitz B. Mapas conceituais: instrumentos didáticos de avaliação e de análise de currículo. São Paulo (SP): Moraes; 1987. 4. Andre MEDAde, Passos LF. Avaliação escolar: desafio e perspectivas. In: Castro AD de, Carvalho AMP, organizadores. Ensinar a ensinar. São Paulo (SP): Pioneira; 2001. p. 177-95.

5. Bogado MLG, Martins JT, Rodrigues IG, Baduy RS, Sato H, Breganó RM. Projeto político pedagógico do curso de enfermagem da Universidade Estadual de Londrina. Londrina (PR): Colegiado do Curso de Enfermagem; 1999. 
6. Martins JT, Dellaroza MSG. O currículo integrado do curso de enfermagem da UEL: mito ou realidade. Anais do Seminário Nacional de Diretrizes para a Educação em Enfermagem no Brasil; 2000; Fortaleza (CE). Fortaleza: ABEn; 2000.

7. Carvalho V. Cuidando, pesquisando e ensinando: acerca de significados e implicações da prática da enfermagem. Rev Latino-am Enfermagem 2004 setembro-outubro; 12(5):806-15.

8. Associação Brasileira de Normas Técnicas. NBR 14724: informação e documentação - trabalhos acadêmicos - apresentação. Rio de Janeiro (RJ): ABNT; 2002.

9. Köche JC. A estrutura dos relatórios. In: Köche JC . Fundamentos de metodologia científica: teoria da ciência e prática da pesquisa. $17^{2} \mathrm{ed}$. Petrópolis (RJ): Vozes; 1997. p.137-51.

10. Polit DF, Hungler BP. Relatórios de pesquisa: leitura e revisão da literatura científica. In: Polit DF, Hungler BP. Fundamentos de pesquisa em enfermagem. $3^{\mathrm{a}}$ ed. Porto Alegre (RS): Artes Médicas; 1995. p.4165.

11. Freire P. Educação e conscientização. In: Freire P. Educação como prática da liberdade. $19^{\mathrm{a}}$ ed. Rio de Janeiro (RJ): Paz e Terra; 1989. p.1-26.

12. Matos FG. O título de um trabalho científico: claro, conciso, concreto e criativo. Ci Cultura agosto 1988; 40(8):778-9.

13. Fachin O. Conclusão. In: Fachin O. Fundamentos de metodologia. $3^{\mathrm{a}}$ ed. São Paulo (SP): Saraiva; 2001. p. 165-6.

14. Demo P. Pesquisa: princípio científico e educativo. $7^{\mathrm{a}}$ ed. São Paulo (SP): Cortez; 2000. 